\title{
Mortality among older patients admitted to the medical wards of Groote Schuur Hospital, Cape Town, South Africa, 2010 - 2013
}

\author{
L A Adebusoye, ${ }^{1}$ MBBS, FWACP, FMCFM, MSc (Epidemiology), DGM (SA); $\mathbf{S}$ Z Kalula, ${ }^{2} \mathrm{MB}$ ChB, PhD, FRCP \\ ${ }^{1}$ Chief Tony Anenih Geriatric Centre, University College Hospital, Ibadan, Nigeria \\ ${ }^{2}$ Albertina and Walter Sisulu Institute of Ageing in Africa, Department of Medicine, Faculty of Health Sciences, University of Cape Town, \\ South Africa
}

Corresponding author: L A Adebusoye (larrymacsoye@yahoo.com)

\begin{abstract}
Background. Geriatric medicine is an evolving specialty in Africa, and little is known about mortality among older patients admitted to medical wards.

Objectives. To determine mortality rates and associated factors among older medical inpatients.

Methods. Electronic data on patients aged $\geq 60$ years admitted to the medical wards of Groote Schuur Hospital, Cape Town, South Africa, between January 2010 and December 2013 were analysed. Data extracted included sex, age, causes of death, and length of stay from date of admission to discharge or death. Results of laboratory tests carried out during the admission were also obtained.

Results. In all, 11254 older patients were admitted (mean (standard deviation) age 70.7 (7.9) years). There were 1701 deaths (15.1\%). The unadjusted mortality rate was 29.6 deaths per 1000 patient-days (PD). The majority (87.5\%) were admitted as emergency cases. Mortality in the first 24 hours was 32.4 deaths per $1000 \mathrm{PD}$. There was a significant increase in mortality with increasing age $(p<0.001)$. Stroke was the commonest cause of mortality (14.5\%). The predictors of mortality were short length of stay on admission (odds ratio (OR) $1.047,95 \%$ confidence interval (CI) 1.033 - 1.061), high white blood cell count (OR 1.064, 95\% CI 1.054 - 1.074), low platelet count (OR 0.999, 95\% CI 0.996 - 1.000), low haemoglobin (OR 0.940, 95\% CI 0.917 - 0.964) and high blood urea (OR 1.042, 95\% CI 1.033 - 1.051).

Conclusions. Mortality among older medical inpatients was high. Modifiable predictors of mortality, especially related to laboratory derangements, should be identified and addressed promptly.
\end{abstract}

S Afr Med J 2019;109(2):116-121. DOI:10.7196/SAMJ.2019.v109i2.13275

Older persons constitute a unique subgroup of adults in any population, not only due to advanced age but also a variety of physiological, social and demographic changes. ${ }^{[1]}$ With increasing age, a number of physiological variables decline substantially, compromising the individual's ability to respond to pathological insults. ${ }^{[2]}$ The determinants of mortality in older persons are numerous and can be grouped as non-modifiable or modifiable factors. ${ }^{[3]}$

Globally, hospital admission and mortality rates among older persons are continually on the increase. ${ }^{[4]}$ This is partly due to the increase in the number of older persons in the population. Hospitalisation for medical illnesses is considered to be a risk factor for death among older persons. ${ }^{[5]}$ Furthermore, such illness usually has adverse effects on health and may lead to negative outcomes such as disability, loss of independence and autonomy, and social isolation. In addition, older patients have increased susceptibility to iatrogenic conditions and nosocomial as well as other infections. ${ }^{[3]}$ Research has shown that older patients accounted for $11.0-56.6 \%$ of all adult hospital medical ward admissions, ${ }^{[5]}$ with a mortality rate of $26.6-57.6 \%{ }^{[5]}$ In Africa, however, the mortality rate is expected to be higher owing to a lack of standard medical facilities.

Globally, older persons are the fastest-expanding age group, with approximately one million people attaining the age of 60 years every month. ${ }^{[3]}$ It is estimated that by the year 2025, eight of the 11 countries with the biggest populations of older persons will be developing countries. ${ }^{[3]}$ The 2014 report on the profile of persons aged $\geq 60$ years in South Africa (SA) showed a population of 4.1 million, representing $8.0 \%$ of the total population. ${ }^{[6,7]}$ This makes SA the African country with the second-largest proportion of older persons (after Mauritius). ${ }^{[6,7]}$ Furthermore, the number of persons aged $\geq 60$ years in SA is expected to increase over 100 -fold from 2.47 million in 1985 to 5.23 million by the year $2025 .^{[6]}$ The projected increase is huge compared with the estimated growth of $74-83 \%$ among young adults. ${ }^{[6,7]}$

\section{Objectives}

Old age is associated with both health and functional decline and the associated increased prevalence of chronic diseases with increased morbidity and hospitalisation. There is, however, a paucity of information on the mortality rate among hospitalised older patients in medical wards in Africa. Most of the existing information on older patients has been extracted or extrapolated from data on general adult patients admitted to medical wards in Africa, ${ }^{[5]}$ which revealed higher levels of hospitalisation and mortality among older persons compared with the young population. ${ }^{[5,6]}$ This study therefore sought to determine mortality rates, temporal trends and associated factors among older patients admitted in a large tertiary hospital in SA.

\section{Methods}

This was a retrospective study of all medical admissions of patients aged $\geq 60$ years between 1 January 2010 and 31 December 2013 at Groote Schuur Hospital (GSH), Cape Town, SA. GSH became 
operational in 1938 and functions as a tertiary and quaternary care hospital. The division of geriatric medicine is one of 18 clinical divisions in the Department of Medicine. Although the hospital has a division of geriatric medicine, there are no beds dedicated to geriatric medicine for acute admissions. Older patients requiring acute medical management are admitted to general medical wards together with other adult medical patients.

Electronic records of all older patients were obtained via the Clinicom Patient Administration System with approval from the chief operations officer of GSH and the Human Research Ethics Committee of the Faculty of Health Sciences at University of Cape Town (ref. no. 012/2015). All older patients who were admitted to the medical wards during the study period were included. We obtained information on their sex, age, length of stay, causes of death (as reported on the basis of clinical impression), and dates of admission and discharge. Laboratory data, which included complete blood counts and urea, creatinine and glucose levels, were obtained from the National Health Laboratory Service (NHLS) data warehouse. These are the investigations most routinely done on acute admission to the hospital. Admission details and laboratory data were linked by means of the patient's unique hospital number. Confidentiality of data was maintained with the exclusion of names and hospital numbers from the analysis, leaving only the serial numbers allotted to the patients.

Data were analysed using the Statistical Package for the Social Sciences version 22 (IBM, USA). Descriptive and inferential statistics were used. Appropriate charts were used to illustrate categorical variables. Unadjusted mortality rates and rate ratios were calculated in patient-days (PD) with $95 \%$ confidence intervals (CIs). The Cochrane-Armitage test for temporal trend was used to test for linearity of the mortality trend over the study period. Logistic regression analysis was used to explore factors that were significantly associated with mortality, and the level of significance was set at $p<0.05$.

\section{Results}

A total of 11254 older patients were admitted to the medical wards during the study period (males $n=5089$, females $n=6$ 165). The mean (standard deviation (SD)) age of the patients was 70.7 (8.0) years (females v. males 71.7 (8.3) years v. 69.5 (7.4) years) ( $t=14.580$; $p<0.001)$. The total number of deaths was 1701 (15.1\%), with 802 (15.8\%) and 899 (14.6\%) deaths occurring among males and females, respectively. The mean (SD) age of males who died was significantly higher than the mean age of males who were discharged, at 70.3 (7.6) years v. 69.4 (7.4) years $(t=3.20 ; p=0.012)$. A similar trend was observed among females, with ages 72.5 (8.5) years v. 71.5 (8.2) years $(t=4.35 ; p<0.0001)$. The majority $(87.5 \%)$ of the patients were admitted as emergency cases. Mortality in the first 24 hours of admission was 32.4 deaths (95\% CI 29.2 - 35.9) per $1000 \mathrm{PD}$, which was not significantly higher among the males at 35.4 deaths $(95 \%$ CI 30.5 - 40.8) per $1000 \mathrm{PD}$ compared with the females at 30.0 deaths (95\% CI 25.9 - 34.6) per 1000 PD ( $p=0.11)$.

Overall, the unadjusted mortality rate was 29.6 (95\% CI 28.2 - 31.0) per 1000 PD. The mortality rate was highest in $2010(31.8$ (95\% CI 28.9 - 34.8) per $1000 \mathrm{PD}$ ) and decreased steadily over the years to 27.0 (95\% CI 24.4 - 29.7) per $1000 \mathrm{PD}$ in 2013. Table 1 shows the unadjusted mortality rates from January 2010 to December 2013 by gender and age groups. Overall, the mortality rate was higher among men compared with women, without a statistical difference (30.3 per 1000 PD v. 29.0 per 1000 PD; $p=0.40$ ). However, the only significant difference between mortality rate and gender was observed in the year 2010 .

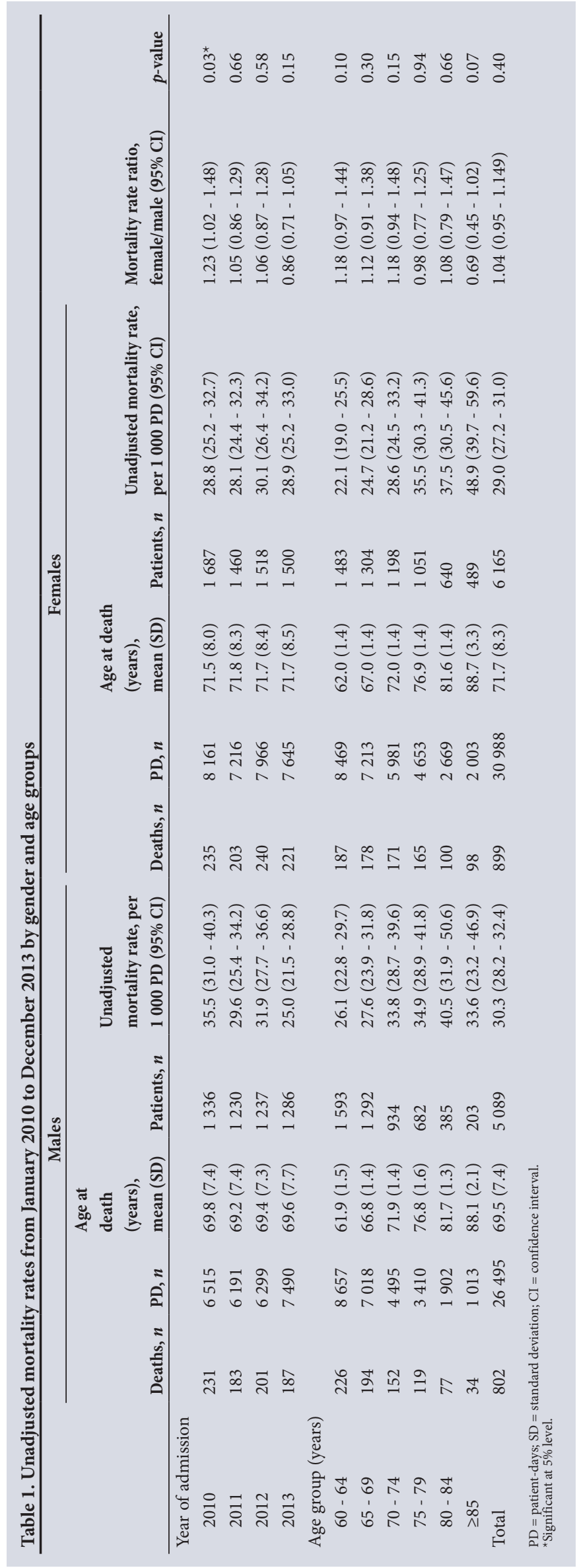


Fig. 1 describes the overall unadjusted mortality rates by age group. There was a significant increase in the mortality rate with age from 24.1 per $1000 \mathrm{PD}$ in the 60 - 64-year age group to 43.8 per $1000 \mathrm{PD}$ in patients aged $\geq 85$ years (Cochrane-Armitage test for linear trend: $\left.\chi^{2}=64.57 ; p<0.0001\right)$.

Diagnoses were obtained in $6143(54.6 \%)$ of the patients. As shown in Table 2, the commonest reasons for admission were myocardial infarction (19.6\%), heart failure (7.4\%) and stroke (7.3\%). However, the highest proportions of deaths were found in patients with stroke
(14.5\%), heart failure (7.9\%) and myocardial infarction (7.2\%). Fig. 2 describes the classification of the diagnoses and proportion of deaths according to the International Classification of Primary Care, 2nd edition (ICPC-2) ${ }^{[8]}$ The highest proportion of deaths were related to the general body system $(22.2 \%)$, while no death was related to the psychological and eye systems.

Table 3 shows the laboratory values of the patients by mortality. Mean white blood cell counts and serum creatinine, blood urea and blood glucose levels were significantly higher among patients who

Table 2. Morbidity pattern by proportion of deaths

\begin{tabular}{|c|c|c|c|}
\hline Morbidities & Died $(N=455), n(\%)$ & Discharged $(N=5688), n(\%)$ & Total $(N=6143), n(\%)$ \\
\hline Myocardial infarction & $33(7.2)$ & $1168(20.5)$ & $1201(19.6)$ \\
\hline Heart failure & $36(7.9)$ & $416(7.3)$ & $452(7.4)$ \\
\hline Stroke & $66(14.5)$ & $385(6.8)$ & $451(7.3)$ \\
\hline Chronic obstructive pulmonary disease & $22(4.8)$ & $335(5.9)$ & $357(5.8)$ \\
\hline Hypertension & $15(3.3)$ & $243(4.3)$ & $258(4.2)$ \\
\hline Pneumonia & $31(6.8)$ & $222(3.9)$ & $253(4.1)$ \\
\hline Ischaemic heart disease & $13(2.9)$ & $172(3.0)$ & $185(3.0)$ \\
\hline Atrioventricular block & $7(1.5)$ & $175(3.1)$ & $182(3.0)$ \\
\hline Paroxysmal tachycardia & $10(2.2)$ & $169(3.0)$ & $179(2.9)$ \\
\hline Delirium & $7(1.5)$ & $162(2.8)$ & $169(2.8)$ \\
\hline Disorders of digestive system (unspecified) & $15(3.3)$ & $145(2.5)$ & $160(2.6)$ \\
\hline Mental disorder (unspecified) & 0 & $153(2.7)$ & $153(2.5)$ \\
\hline Cardiac disease (unspecified) & $6(1.3)$ & $142(2.5)$ & $148(2.4)$ \\
\hline Dementia & $14(3.1)$ & $127(2.2)$ & $141(2.3)$ \\
\hline Malignancy with metastasis & $31(6.8)$ & $97(1.7)$ & $128(2.1)$ \\
\hline Diabetes mellitus & $4(0.9)$ & $99(1.7)$ & $103(1.7)$ \\
\hline Chronic kidney disease & $13(2.9)$ & $74(1.3)$ & $87(1.4)$ \\
\hline Dermatitis & $4(0.9)$ & $75(1.3)$ & $79(1.3)$ \\
\hline Bronchial asthma & $10(2.2)$ & $62(1.1)$ & $72(1.2)$ \\
\hline Pulmonary tuberculosis & $10(2.2)$ & $52(0.9)$ & $62(1.0)$ \\
\hline Acute renal failure & $16(3.5)$ & $45(0.8)$ & $61(1.0)$ \\
\hline Acute upper respiratory tract infection & $2(0.4)$ & $55(1.0)$ & $57(0.9)$ \\
\hline Epilepsy & $1(0.2)$ & $52(0.9)$ & $53(0.9)$ \\
\hline Peptic ulcer disease & $2(0.4)$ & $50(0.9)$ & $52(0.8)$ \\
\hline Gastroenteritis & $3(0.6)$ & $46(0.8)$ & $49(0.8)$ \\
\hline Disorders of urinary system (unspecified) & $4(0.9)$ & $45(0.8)$ & $49(0.8)$ \\
\hline Septicaemia & $14(3.1)$ & $34(0.6)$ & $48(0.8)$ \\
\hline Rheumatic heart disease & 0 & $45(0.8)$ & $45(0.7)$ \\
\hline Anaemia & $1(0.2)$ & $42(0.7)$ & $43(0.7)$ \\
\hline Non-rheumatic heart disease & $1(0.2)$ & $39(0.7)$ & $40(0.7)$ \\
\hline Pulmonary heart disease & 0 & $38(0.7)$ & $38(0.6)$ \\
\hline Atrial fibrillation & $1(0.2)$ & $36(0.6)$ & $37(0.6)$ \\
\hline Cellulitis & $2(0.4)$ & $32(0.6)$ & $34(0.6)$ \\
\hline Chronic liver disease & $4(0.9)$ & $30(0.5)$ & $34(0.6)$ \\
\hline Psoriasis & 0 & $28(0.5)$ & $28(0.5)$ \\
\hline Cardiomyopathy & $1(0.2)$ & $25(0.4)$ & $26(0.4)$ \\
\hline Phlebitis & 0 & $20(0.4)$ & $20(0.3)$ \\
\hline Systemic poisoning & $2(0.4)$ & $16(0.3)$ & $18(0.3)$ \\
\hline Psychosis & 0 & $18(0.3)$ & $18(0.3)$ \\
\hline Transient ischaemic attack & $1(0.2)$ & $17(0.3)$ & $18(0.3)$ \\
\hline Neuropathies & 0 & $16(0.3)$ & $16(0.3)$ \\
\hline Multiple myeloma & $2(0.4)$ & $13(0.2)$ & $15(0.2)$ \\
\hline Arterial diseases & 0 & $13(0.2)$ & $13(0.2)$ \\
\hline Metabolic imbalance & 0 & $13(0.2)$ & $13(0.2)$ \\
\hline Parkinson's disease & 0 & $13(0.2)$ & $13(0.2)$ \\
\hline Ulcerative colitis & $1(0.2)$ & $12(0.2)$ & $13(0.2)$ \\
\hline Others & $50(11.0)$ & $422(7.4)$ & $472(7.9)$ \\
\hline
\end{tabular}


died during the hospital admission compared with patients who were discharged. The mean haemoglobin concentration was significantly lower in patients who died compared with patients who were discharged. Although notably lower platelet counts were observed among patients who died than in those who survived, this finding was not statistically significant.

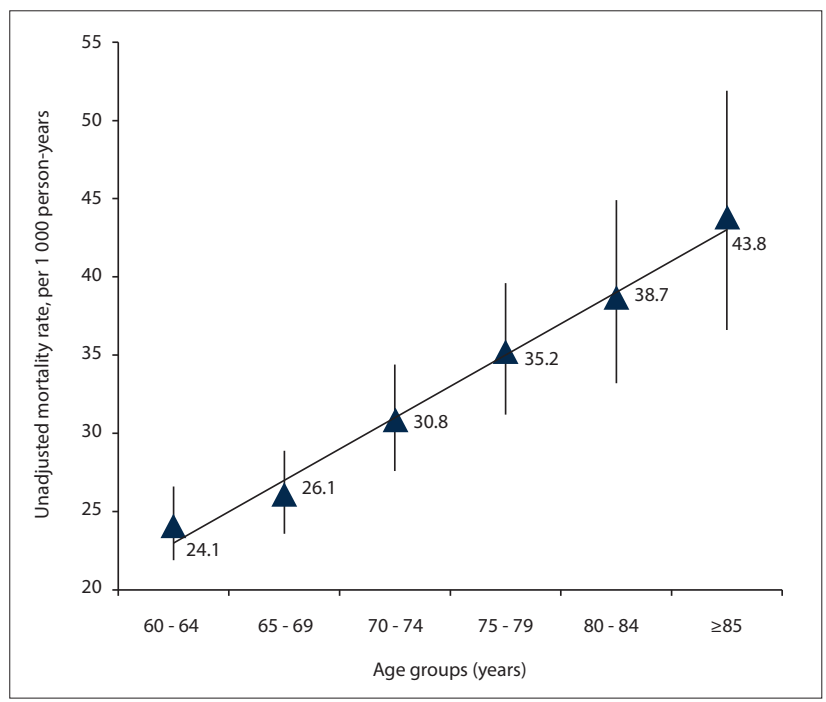

Fig. 1. Unadjusted mortality rate by age groups (Cochrane-Armitage test for linear trend: $\chi^{2}=64.57 ; \mathrm{p}<0.0001$ ).



Fig. 2. Diagnoses according to the International Classification of Primary Care, 2nd edition, ${ }^{[8]}$ by proportion of deaths.
Table 4 presents a logistic regression analysis of the factors associated with mortality. The logistic model was found to be statistically significant $\left(\chi^{2}(8)=642.994 ; p<0.0001\right)$. The model explained $13.5 \%$ (Nagelkerke $R^{2}$ ) of the variance in mortality. It also correctly classified $84.2 \%$ of cases. The most significant factors associated with mortality were short length of stay on admission (odds ratio (OR) 1.047, 95\% CI 1.033 - 1.061; $p<0.0001$ ), high white blood cell count (OR 1.064, $95 \%$ CI $1.054-1.074 ; p<0.0001$ ), low platelet count (OR 0.999, $95 \%$ CI $0.996-1.000 ; p=0.006)$, low haemoglobin concentration (OR 0.940, 95\% CI $0.917-0.964 ; p<0.0001$ ) and high blood urea level (OR 1.042, 95\% CI 1.033 - 1.051; $p<0.0001$ ).

\section{Discussion}

Factors associated with increased mortality in older patients admitted to medical wards were male sex (on bivariate analysis), high white blood cell count, low haemoglobin concentration, high urea level and a short hospital stay. This study was conducted in a large tertiary hospital in SA and deaths were reported in almost one-sixth (15.1\%) of older patients. This proportion of deaths was similar to that reported in a systematic review of in-hospital mortality among elderly patients in medical wards in Africa ${ }^{[5]}$ and South America. ${ }^{[9]}$ However, mortality was higher than that reported from Europe (8.4\%). ${ }^{[10]}$ Available mortality data on hospitalised older patients from Nigeria $(26.7-31.7 \%)^{[11,12]}$ showed a higher proportion of deaths than the findings of the present study. ${ }^{[5]}$ A previous retrospective study $(2002$ - 2009) carried out in the same setting as our study found a proportion of deaths of $12-17 \%$, although this population included young adults. ${ }^{[13]}$

The proportion of deaths in the present study was higher among males than among females, though without statistical significance. This finding was not unexpected, as male sex has been reported to be an independent risk factor associated with inpatient mortality in medical wards in other studies. ${ }^{[5,13]}$ Other factors that work singly or jointly have been adduced for the mortality difference between male and female older patients. ${ }^{[14]}$ Older females have longer life expectancy, better health-seeking behaviour, better lifestyle habits and less exposure to stress. In addition, cardiocirculatory protection of females due to the effect of hormones during their fertile years is a major contribution. ${ }^{[14]}$

Advancing age has been reported as an immutable risk factor for mortality in hospitalised older patients, and our observation was no different. ${ }^{[5,13,15]} \mathrm{A}$ significant linear increase in mortality rate with increasing age was observed in both sexes. Also, there were some differences across the years of study. A possible explanation for the reduction in mortality between 2010 and 2013 may be the increase in the number of medical beds, which provided space for improved patient assessment. Medical beds were increased from 142 in 2010 to 153 in 2013. Similarly, the medical teams (called firms) were reorganised, resulting in improved efficiency. The medical firms were each provided with a dedicated ward in 2013, whereas in

\begin{tabular}{|c|c|c|c|c|c|}
\hline & $n$ & Dead, mean (SD) & Discharged, mean (SD) & $t$ & $p$ \\
\hline White blood cell count $\left(\times 10^{9} / \mathrm{L}\right)$ & 9522 & $15.0(0.1)$ & $11.2(0.1)$ & 12.713 & $<0.0001^{*}$ \\
\hline Platelet count $\left(\times 10^{9} / \mathrm{L}\right)$ & 8758 & $311.2(0.2)$ & $312.0(0.1)$ & -0.019 & 0.985 \\
\hline Haemoglobin (g/dL) & 9559 & $11.5(2.9)$ & $12.3(2.6)$ & -9.194 & $<0.0001^{*}$ \\
\hline Serum creatinine $(\mathrm{mmol} / \mathrm{L})$ & 10519 & $219.8(26.4)$ & $134.1(15.3)$ & 12.569 & $<0.0001^{*}$ \\
\hline Urea $(\mathrm{mmol} / \mathrm{L})$ & 9382 & $17.6(1.6)$ & $10.7(0.9)$ & 15.804 & $<0.0001^{*}$ \\
\hline Blood glucose $(\mathrm{mmol} / \mathrm{L})$ & 365 & $11.2(0.9)$ & $8.2(0.7)$ & 2.077 & $0.042^{*}$ \\
\hline
\end{tabular}


Table 4. Logistic regression analysis of the factors associated with mortality

\begin{tabular}{llllll}
\hline & & & & \multicolumn{2}{c}{$\mathbf{9 5 \%}$ CI for OR } \\
\cline { 4 - 6 } Variables & Beta coefficient & $p$-value & OR & Lower & Upper \\
\hline Age & 0.004 & 0.318 & 1.004 & 0.996 & 1.012 \\
Length of stay & 0.046 & $<0.0001^{*}$ & 1.047 & 1.033 & 1.061 \\
White cell count & 0.001 & $<0.0001^{*}$ & 1.064 & 1.054 & 1.074 \\
Platelet count & -0.001 & $0.006^{*}$ & 0.999 & 0.996 & 1.000 \\
Haemoglobin & -0.062 & $<0.0001^{*}$ & 0.940 & 0.917 & 0.964 \\
Creatinine & 0.002 & 0.366 & 1.000 & 0.999 & 1.000 \\
Urea & 0.041 & $<0.0001^{*}$ & 1.042 & 1.033 & 1.051 \\
OR =odds ratio; CI = confidence interval. & & & & &
\end{tabular}

previous years, medical wards were shared as an open space by the different medical firms. Having space dedicated to each team may have impacted positively on care of older patients in the medical wards by providing focused patient care and improved handovers. The improvement in mortality occurred despite improved access to tertiary care through the expanded primary healthcare platform. This platform started in 1994 and has improved access to care for older patients with advanced morbidities. ${ }^{[13]}$

The commonest reasons for hospitalisation in this study were myocardial infarction, heart failure and stroke. Acute illnesses such as stroke and acute exacerbation of respiratory disease were common causes of mortality, as has been found in other studies in Africa. ${ }^{[5,16,17]}$ Older people are facing the double burden of communicable and non-communicable diseases as part of the health transition in Africa. ${ }^{[18]}$ Acute exacerbation of chronic diseases, infections and deranged metabolic factors are therefore common reasons for hospitalisation and mortality. ${ }^{[5,16]}$ Not surprisingly, the leading causes of death in this study were septicaemia, acute renal failure and malignancies with metastasis. ${ }^{[5,16,17]}$ These were also reflected in the predictors of mortality found, as the risk of mortality increased by $6.4 \%$ for every $1 \times 10^{9} / \mathrm{L}$ increase in the white blood cell count. Anaemia from causes including malignancies with metastasis was a predictor, as there was a $6.0 \%$ increase in mortality with every $1 \mathrm{~g} / \mathrm{dL}$ decrease in the haemoglobin concentration. Serum creatinine was not found to be a predictor of mortality, although there was a significant association between mortality and serum creatinine level on bivariate analysis. However, blood urea was a predictor, with a $4.2 \%$ increase in mortality with every $1 \mathrm{mmol} / \mathrm{L}$ increase in the urea level. Serum creatinine may not have been an independent predictor of mortality, as creatinine is a poor screening test for renal failure in older patients, who commonly have reduced muscle mass. ${ }^{[19]}$ Increased length of stay has been associated with increased mortality in a number of studies, particularly in patients with delirium. ${ }^{[9]}$ In our study, reduced length of stay was associated with increased mortality. This is an indication of the high mortality rate (32.4 deaths per $1000 \mathrm{PD})$ in the first 24 hours of admission. The high rate is probably due to patients presenting at an advanced stage of the illness, and an inadequate service provided at the primary level of care. ${ }^{[5,9]}$

\section{Study strengths and limitations}

The present study is of significance in that it is among the few studies in Africa to evaluate mortality and associated factors in the older population in Africa. It has limitations in that it was retrospective and a diagnosis was available in the dataset for just over half of the patients. Prehospital functional assessment and nutritional status, which have an impact on mortality in older people, could not be obtained. The study was limited to one centre and is therefore not generalisable to other settings in SA.

\section{Conclusions}

There was a high mortality rate among older patients admitted to the medical wards of a large tertiary hospital in SA, with a male preponderance. The high proportion of patients presenting on an emergency basis calls for effective and more frequent outpatient appointments. This extra effort will facilitate early diagnosis and management of acute exacerbations and complications of morbidities. Lastly, the association of mortality with laboratory biomarkers shows the need for adequate investigation and management of morbidities with which these biomarkers are associated.

\section{Declaration. None.}

Acknowledgements. We thank the patients whose data were used for this study and the hospital management for allowing access to the data. We thank Wendy Bryant for providing the data, the NHLS warehouse for providing the laboratory data, and Samuel Oke-Job and Leon Geffen for assisting with the data analysis.

Author contributions. SZK was the supervisor of the project. LAA and SZK were involved in the project design, writing of the manuscript and conceptual contributions. LAA was responsible for the collection of data and analysis. The manuscript was read and verified by both authors.

Funding. None.

Conflicts of interest. None.

1. Clausen F, Sandberg E, Ingstaad B, Hjortdan P. Morbidity and healthcare utilization among the elderly people in Mmankgodi village, Botswana. J Epidemiol Community Health 2000;54(1):58-63. https:// doi.org/10.1136/jech.54.1.58

2. Cruz-Jentoft AJ, Baeyens JP, Bauer JM, et al. Sarcopenia: European consensus on definition and diagnosis. Report of the European Working Group on Sarcopenia in Older People. Age Ageing 2010;39(4):412-423. https://doi.org/10.1093/ageing/afq034

3. Maia FOM, Duarte YAO, Lebrao ML, Santos JLF. Risk factors for mortality among elderly people. Rev Saude Publica 2006;40(6):1049-1056. https://doi.org/10.1590/S0034-89102006005000009

4. Sanya EO, Akande TM, Opadijo G, Olarinoye Jk, Bojuwoye BJ. Pattern and outcome of medical Sanya EO, Akande TM, Opadijo G, Olarinoye Jk, Bojwwoye Bj. Palten and outcome of medica admission of elderly patients seen at University of Horin Teaching Hospital, Horin. Afr J Med Med Sc 2008;37(4).375 Adebusoye LA, Owolabi MO, Kalula SZ, Ogunniyi A. All-cause mortality among elderly patient admitted to the medical wards of hospitals in Africa: A systematic review. Niger J Health Sci 2015;15(1):45-51. https://doi.org/10.4103/1596-4078.171372

6. Joubert J, Bradshaw D. Population ageing and health challenges in South Africa. In: Steyn K, Fourie J, Temple N, eds. Chronic Diseases of Lifestyle in South Africa: 1995 - 2005. Cape Town: South African Medical Research Council, 2006:204-219. http://www.mrc.ac.za/sites/default/files/files/2016-07-14/ cdl1995-2005.pdf (accessed 16 December 2018).

7. United Nations, Department of Economic and Social Affairs, Population Division. World population ageing 2013. ST/ESA/SER.A/348. http://www.un.org/en/development/desa/population/publications/ pdf/ageing/WorldPopulationAgeing2013.pdf (accessed 16 December 2018).

8. Graeme M, Helena B. ICPC-2-E: The electronic version ICPC-2. Fam Pract 2000;17(5):448. https:// doi.org/10.1093/fampra/17.5.448

9. Silva TJ, Jerussalmy CS, Farfel JM, Curiati JA, Jacob-Filho W. Predictors of in-hospital mortality among older patients. Clinics (Sao Paulo) 2009;64(7):613-618. https://doi.org/10.1590/S180759322009000700002

10. Ayaz T, Serap BS, Osman ZS, Ozlem B, Halil R. Factors affecting mortality in elderly patients hospitalized for nonmalignant reasons. J Aging Res 2014;2014:article ID 534315. https://doi org/10.1155/2014/584315 
11. Onwuchekwa AC, Asekomeh EG. Geriatric admissions in a developing country: Experience from a tertiary centre in Nigeria. Ethn Dis 2009;19(3):359-362. https://www.ncbi.nlm.nih.gov/ pubmed/19769021 (accessed 18 December 2018).

12. Sanya E, Kolo P, Adekeye K, Abiodun A, Olanrewaju T. Profile and causes of mortality among elderly patients seen in a tertiary care hospital in Nigeria. Ann Afr Med 2011:10(4):278-283, https://doi. patients seen in a tertiary care
org/10.4103/1596-3519.87043

13. Myer L, Smith E, Mayosi BM. Medical inpatient mortality at Groote Schuur Hospital, Cape Town, 2002 - 2009. S Afr Med J 2013;103(1):28-35. https://doi.org/10.7196/SAMJ.6285

4. Maia FdeOM, Duarte YAO, Lebrão ML, Santos JLF. [Risk factors for mortality among elderly people]. Rev Saude Publica 2006;40(6):1049-1056. https://doi.org/10.1590/S0034-89102006005000009

15. Statistics South Africa. Mortality and causes of death in South Africa, 2014: Findings from death notification. https://www.datafirst.uct.ac.za/dataportal/index.php/catalog/566/download/7869 (accessed 18 December 2018)

16. Belayachi J, Dendane T, Madani N, Abidi K, Abouqal R, Zeggwagh AA. Factors predicting mortality in elderly patients admitted to a Moroccan medical intensive care unit. S Afr I Crit Care 2012:28(1):22-27. https://doi.org/10.7196/SAJCC.122
17. Naidoo A. Trends in adult medical admission at Tambo Memorial Hospital Gauteng between 2005 and 2007. Unpublished research report submitted to the Faculty of Health Sciences, University of Witwatersrand, Johannesburg, in partial fulfilment of the requirements for the degree of Masters in Public Health. http://hdl.handle.net/10539/8795 (accessed 7 January 2019).

Public Health. http://hdl.handle.net/10539/8795 (accessed 7 January 2019).
8. Maher D, Smeeth L, Sekajugo J. Health transition in Africa: Practical policy proposals for primary care. 18. Maher D, Smeeth L, Sekajugo J. Health transition in Africa: Practical policy proposals for
Bull World Health Organ 2010;88(12):943-948. https://doi.org/10.2471/BLT.10.077891

19. Swedko PJ, Clark HD, Paramsothy K, Akbari A. Serum creatinine is an inadequate screening test for renal failure in elderly patients. Arch Intern Med 2003; 63(3):356-360. https://doi.org/10.1001/ archinte.163.3.356

Accepted 5 July 2018. 